\title{
Secure Crime Case Summary in Police Station Using Block Chain Technology
}

\author{
Surya Tej KR, Poornima N
}

${ }^{1}$ Department of Computer Science and Engineering, The National Institute of Engineering, Mysuru, Karnataka, India

${ }^{2}$ Assistant Professor, Department of Computer Science and Engineering, The National Institute of Engineering, Mysuru, Karnataka, India

Article Info

Volume 7, Issue 4

Page Number: 50-53

Publication Issue :

July-August-2021

\section{Article History}

Accepted : 01 July 2021

Published : 06 July 2021

\section{ABSTRACT}

The data owner may adapt attribute-based encryption to encrypt the stored information for attaining access control and keeping data secure, in the cloud. As a solution to this, an encryption-based algorithm with delegation can be used. Therefore, AES Rijndael algorithm is adapted to encrypt and decrypt the data using the same key with the digitization of traditional records, police stations encounter difficult problems, such as crime case summary storage and access. Managing department, spends considerable time querying the required data when accessing crime case summary. On this basis, this study proposes a case summary sharing scheme which uses ciphertext-based encryption to ensure data confidentiality and access control of crime case summary The officer may encrypt the stored information for attaining access control and keeping data secure. Therefore, AES Rijndael algorithm is used for encryption. This algorithm ensures security of information and enables Privacy.

Keywords : Android application, Training and Placement Department, Firebase, Angular

\section{INTRODUCTION}

Cloud storage is a cloud computing model that stores data on the Internet through a cloud computing provider who manages and operates data storage as a service.

It's delivered on demand with just-in-time capacity and costs, and eliminates buying and managing your own data storage infrastructure. With cloud storage, there is no hardware to purchase, storage to provision, or capital being used.

Cloud storage allows IT to quickly deliver the exact amount of storage needed, right when it's needed. This allows IT to focus on solving complex application problems instead of having to manage storage systems. 
Blockchain decentralization eliminates the concentration of cloud storage servers and solves the security flaws caused by network attacks

\section{LITERATURE SURVEY}

The concept of blockchain first introduced by Satoshi Nakamoto in 2008 in his paper which is later implemented by Bitcoin blockchain. The paper titled "Bitcoin: A Peer-to-Peer Electronic Cash System" discuss P2P electronic cash system which will allow online payment to be sent directly from one party to another without third-party involvement like Banks. The trust issue was resolved using digital signatures, and a solution was provided to another critical issue with Electronic cash system called double-spending in the form of P2P network. The network timestamps transactions by hashing them to an ongoing chain of hash-based proof-of-work, forming a record that cannot be changed. The longest chain serves as proof of the sequence of events witnessed and that it came from the most significant pool of CPU power. The longest chain cannot be generated by attackers until they own more than 50 percent of CPU power. Messages are broadcast on a best effort basis, and nodes can leave and rejoin the network at will, accepting the longest proof-of-work chain [Nak08].Blockchains are shared, immutable ledgers for recording the history of transactions. They foster a new generation of transactional applications that establish trust, accountability, and transparency.

The primary motive of blockchain development was initially for financial application but after the introduction of smart contract, blockchain applications bear no boundary anymore. Blockchain can now be used as for financial and non-financial applications [CNSK15]. Among one of these applications is IoT. Blockchain allow us to have a distributed P2P network where trustless members can interact with each other without a trusted mediator, in a provable way. Smart contracts-scripts that reside on the blockchain that allows automation of multi-step processes. Blockchain facilitates the sharing of services and resources leading to the creation of a marketplace of services between devices and allows us to automate in a cryptographically verifiable manner several existing, time-consuming workflows. The blockchain-IoT combination is powerful and can cause significant transformations across several industries, paving the way for new business models and novel, distributed applications [CD16]. In the IoT era, new connected devices will spread highly sensitive personal data. Sending this type of data to centralized system represents a severe risk to privacy. A possible solution to protect privacy is to leverage the use of Peer-to-Peer storage networks in combination with the blockchain. However, such architecture, despite promising, embeds still limitations, especially regarding scalability [CVM17]. There are three significant value propositions provide by blockchain based IoT platform given in [OCo17].

1.Build trust between the parties that transact together. Blockchain-based IoT enables devices to participate in transactions as a trusted party. Individuals in a deal may not trust each other but unmodifiable data from devices stored on blockchain provide the necessary trust for businesses and people to cooperate.

2.Reduce costs which enable participants to reduce monetary and time commitment costs by eventually removing the 'middle man' from the process. Transactions and device data are now displayed on a peer to peer basis, removing most legal or contractual costs.

3.Accelerate transactions which enable more transactions overall because the

'middle man' is removed from the process. Smart contracts allow organizations to reduce the time needed for completing legal or contractual responsibilities. 
Blockchain not only gives the solution to trust, reduce cost, accelerate the transaction, protect privacy but also give decentralize storage, accessibility to data on the blockchain. Which provides a solution to decentralize management of data, digital property resolution which having the vital impact on how big data may evolve [KM17].

\section{SYSTEM ANALYSIS}

\section{A. EXISTING SYSTEM}

In the existing system, people go to police stations to complain against the crimes faced by them.

These complaints are registered by the police and they maintain the crime case summary and store it in the database. Whenever there is need of these files, they retrieve it from the database.

These files can be accessed by any hacker hence there is no security for these files. There should be a solution adapted to increase the security of crime case summary.

\section{B. PROPOSED SYSTEM}

$\checkmark$ We propose a system to develop a web application which will help to secure the crime case summary registered in the police stations.

$\checkmark \quad$ The police officer files crime case summary and stores it in the database. Each case summary is encrypted by using AES Rijndael algorithm and is stored as block chain in the database.

$\checkmark$ Access key is generated and notification is sent to the police officer for verification.

$\checkmark \quad$ Officer of that particular police station can view the crime case summary by decrypting the blocks using key. This enables privacy and security and prevents from third- party access.

\section{SYSTEM DESIGN}

The architecture system consists of three modules:

1. Application manager
2. Police station

3. Higher officer

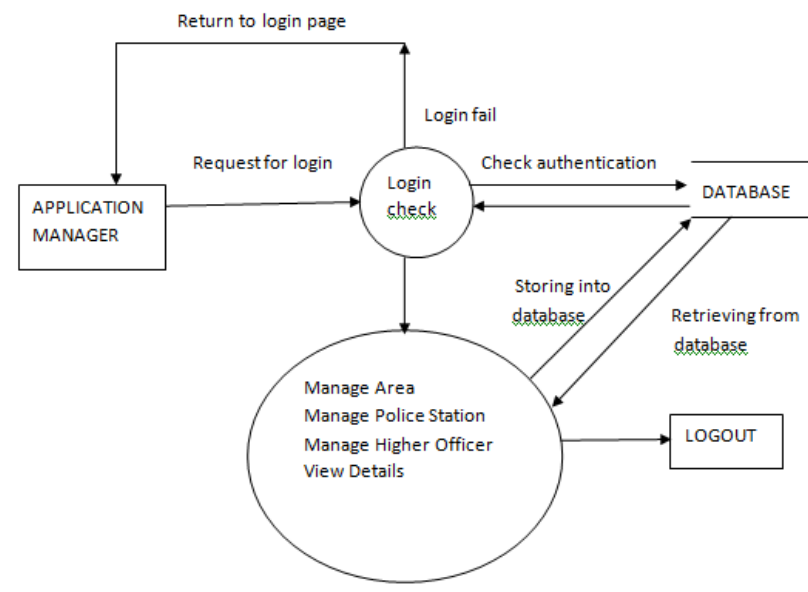

Fig. 1 - Application manager Flow Diagram placements can be an exhausting task.

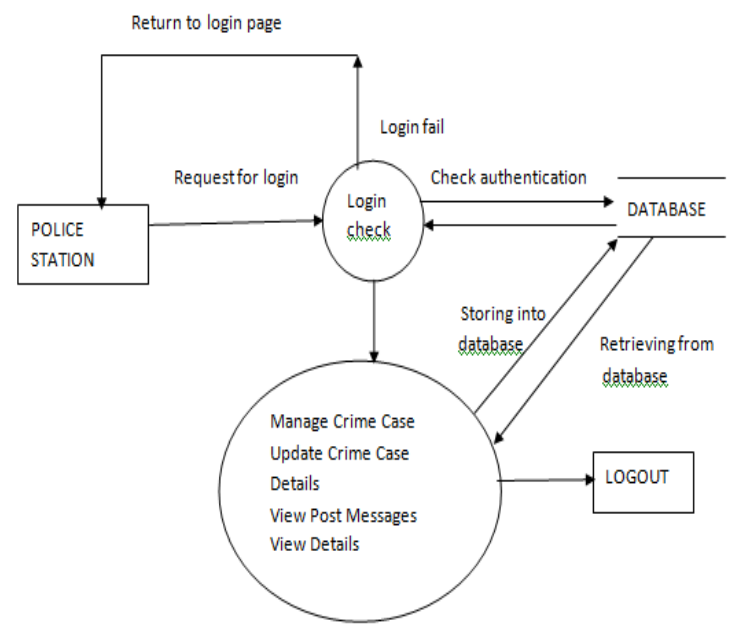

Fig. 2 - Police station Flow Diagram

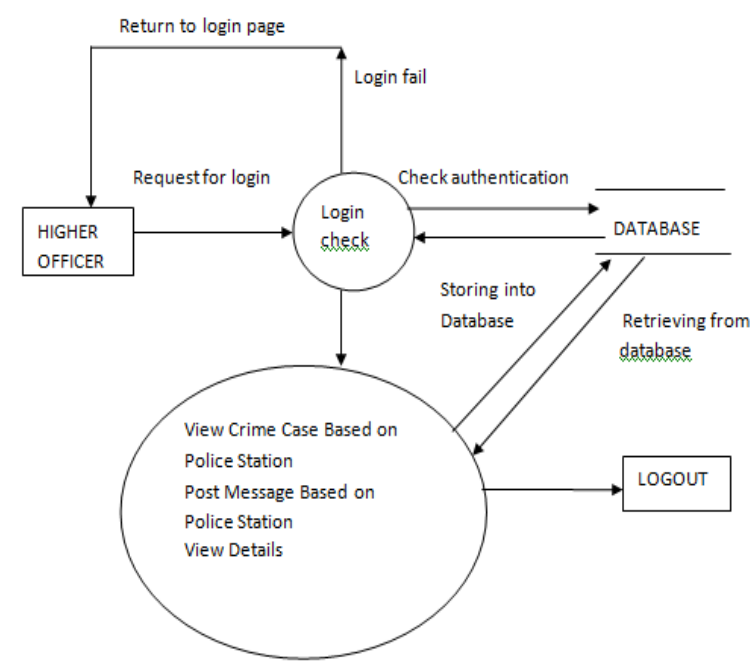

Fig. 3 - Higher Officer Flow Diagram 


\section{SYSTEM IMPLEMENTATION}

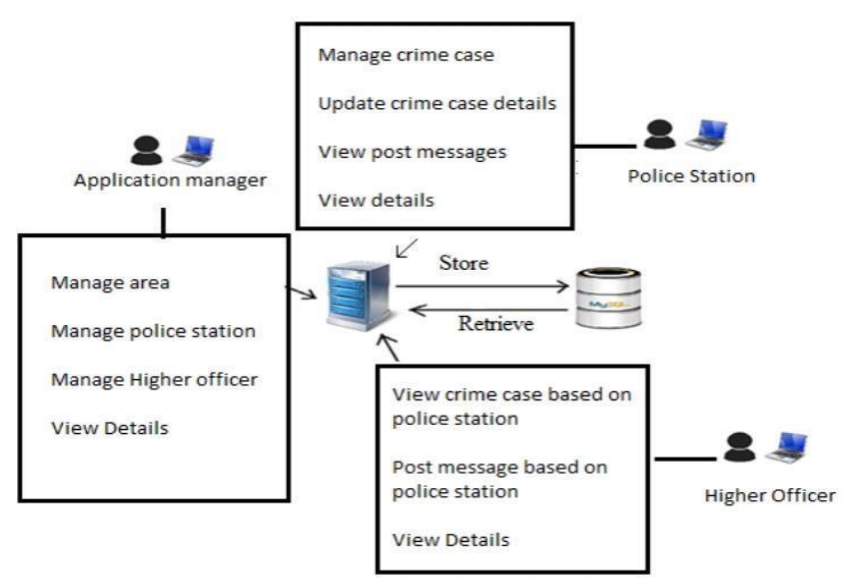

Fig. 4 - System Implementation Diagram

\section{CONCLUSION}

The proposed system developed is a web application which will help to secure the crime case summary registered in the police stations. This application will give security for the crime case details using block chain technology by incorporating AES Rijndael Algorithm \& QRCode Image.

\section{REFERENCES}

[1]. G. Irving and J. Holden, "How blockchaintimestamped protocols could improve the trustworthiness of medical science.," F1000Research, vol. 5, no. May, p. 222, 2016.

[2]. K. Croman et al., "On scaling decentralized blockchains (A positionpaper)," Lect. Notes Comput. Sci. (including Subser. Lect. Notes Artif.

[3]. Pro C\# with .Net 3.0 platform :- Andrew Troelsem

[4]. Professional ASP .NET 3.5:-Bill Evjen,Scott Hanselman,Devin Rader

[5]. Fundamentals of Database Systems:- Elmasri \& B Navathe.
[6]. Programming in C\# A primer 3rd edition:- E Balagurusamy.

[7]. Ian Somerville -"Software Engineering"-8th edition, Pearson Education Press.

[8]. http://www.w3schools.com/aspnet/

[9]. http://msdn.microsoft.com/en-us/aa336522

[10]. http://www.learnvisualstdio.net/asp.net2.0

\section{Cite this article as :}

Surya Tej KR, Poornima N, "Secure Crime Case Summary in Police Station Using Block Chain Technology ", International Journal of Scientific Research in Computer Science, Engineering and Information Technology (IJSRCSEIT), ISSN : 24563307, Volume 7, Issue 4, pp.50-53, July-August-2021. Available at

doi : https://doi.org/10.32628/CSEIT217410

Journal URL : https://ijsrcseit.com/CSEIT217410 